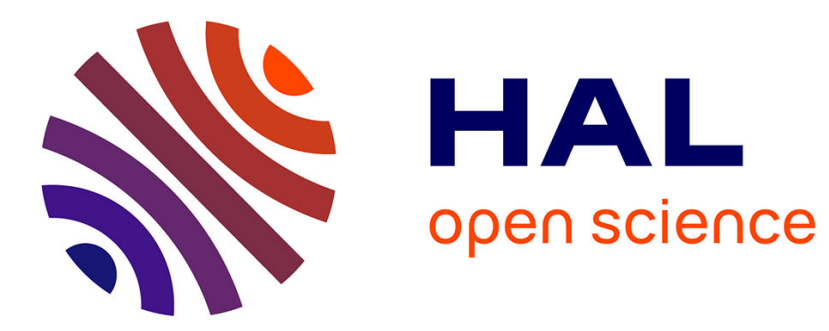

\title{
Ajustements agricoles et structuration de réseaux marchands entre la campagne et la ville en Afrique de l'Est

\author{
Sylvain Racaud
}

\section{- To cite this version:}

Sylvain Racaud. Ajustements agricoles et structuration de réseaux marchands entre la campagne et la ville en Afrique de l'Est. Pour, revue du Groupe Ruralités, Éducation et Politiques, 2014, 221, pp.191-196. hal-02322835

\section{HAL Id: hal-02322835 \\ https://hal.science/hal-02322835}

Submitted on 21 Oct 2019

HAL is a multi-disciplinary open access archive for the deposit and dissemination of scientific research documents, whether they are published or not. The documents may come from teaching and research institutions in France or abroad, or from public or private research centers.
L'archive ouverte pluridisciplinaire HAL, est destinée au dépôt et à la diffusion de documents scientifiques de niveau recherche, publiés ou non, émanant des établissements d'enseignement et de recherche français ou étrangers, des laboratoires publics ou privés. 
Sylvain Racaud

Chercheur associé au laboratoire Dynamiques Rurales, Université de Toulouse 2 Le Mirail,

Post-doc Labex SMS.

Ajustements agricoles et structuration de réseaux marchands entre la campagne et la ville en Afrique de l'Est

À partir de 2007, ce qui a été qualifié d' «émeutes de la faim » dans des pays des Suds a mis en avant la problématique de la faim d'une manière inhabituelle, les images des journaux télévisés ne montraient pas des réfugiés parqués dans des camps ou des pauvres villageois décharnés aux pieds nus, mais des individus de classes moyennes urbaines ${ }^{1}$. Ces évènements s'inscrivent dans un mouvement de forte volatilité des prix des matières premières et en particulier après la forte et soudaine hausse des prix des céréales en $2007^{2}$. Ces faits soulignent l'ampleur de la dépendance de l'approvisionnement alimentaire des citadins aux marchés mondiaux. Pour autant, le ravitaillement en denrées s'appuie également sur les agricultures nationales. Les villes africaines sont connectées de manières plus ou moins intenses et variées à leurs arrières pays, à travers l'attachement des urbains pour leur village ou la structuration de réseaux marchands entre les zones de production et celles de consommation. L'agriculture réalisée dans les campagnes dotées d'avantages comparatifs ${ }^{3}$ telles celles du périrurbain ou celles des montagnes sont intégrées au système d'approvisionnement des villes. Cette contribution présente, en soulignant le rôle du réseau marchand, comment s'organise une agriculture à destination des marchés urbains estafricains.

Essor d'une agriculture alimentaire commerciale destinée aux marchés urbains

Les idées reçues sur l'incapacité des campagnes africaines à nourrir les villes ont longtemps prévalu et s'expliquent en grande partie par des choix politiques en matière de développement. Les États ont privilégié les importations massives de céréales pour satisfaire la demande urbaine et garantir des prix bas mais aussi implicitement afin de prévenir les éventuelles agitations proches des lieux du pouvoir politique, d'autant plus dans une situation de croissance effrénée des métropoles africaines à partir des années 1960. Dans ce contexte,

\footnotetext{
${ }^{1}$ D'autres causes profondes («aspirations démocratiques », chômage structurel, etc.) expliquent également ces émeutes.

${ }^{2}$ La croissance des prix repose sur de nombreux facteurs parmi lesquels : baisse des stocks mondiaux, homogénéisation des pratiques alimentaires qui provoque un détournement des protéines végétales vers la production de protéines animales afin de satisfaire la demande accrue en viande, aléas climatiques, développement des biocarburants, orientation des agricultures des pays des Suds vers l'exportation au détriment des cultures vivrières, crise financière et augmentation de la spéculation sur les matières premières agricoles, etc. ${ }^{3}$ Le principe des avantages comparatifs repose sur l'idée que les échanges sont réalisés en fonction de la comparaison entre les atouts et les coûts de production des différents partenaires ou territoires. Les conditions environnementales (l'abondance de ressources naturelles) confèrent aux montagnes des atouts spécifiques et la proximité avec le centre de consommation offre à l'agriculture périurbaine des avantages par rapport à celle des campagnes plus lointaines.
} 
les grandes villes ont été perçues comme des «vampires » vidant les campagnes de leurs forces vives, accaparant les productions agricoles et empêchant le développement rural.

Cependant, cette vision dichotomique urbain/rural sur laquelle se sont d'ailleurs appuyés les programmes des grandes agences internationales de développement (Charlery de la Masselière, 2005) est aujourd'hui remise en cause et la ville est désormais présentée, même par les bailleurs de fonds internationaux, comme un moteur de l'essor des campagnes, comme un facteur structurant d'une agriculture vivrière marchande.

L'agriculture d'exportation et l'agriculture vivrière ont coexisté au sein des mêmes unités de production et leur rapport a changé, souvent au bénéfice des secondes, après les bouleversements de l'environnement économique liés à la libéralisation des filières d'exportation (cacao, café, coton, thé, etc.) dans les années 1990 (Chaléard, 1996). Ainsi, un cadre inédit de production et de mise en marché des produits agricoles a favorisé l'essor d'une agriculture vivrière marchande en direction de la demande urbaine. Ces reconversions ont été très importantes dans les terres d'élections des cultures traditionnelles d'exportation, comme les montagnes et les hautes terres. Ces zones d'altitude, anciennement intégrées à des réseaux commerciaux de longs cours, bénéficient de conditions environnementales favorables pour la fourniture en quantité et en variété de vivres.

Les agriculteurs ont alors modifié leurs systèmes de production au niveau de la composition des cultures, de l'usage des intrants, des techniques, de la main d'œuvre, de la gestion du sol et des modes de commercialisation. Du fait des densités humaines importantes, les superficies cultivées par unité de production sont très petites, elles sont en moyenne de 0,9 hectares sur les pentes du mont Rungwe dans le sud-ouest tanzanien, où les densités peuvent atteindre 400 habitants au km² (Racaud, 2013). Les producteurs ont privilégié les denrées à destination des marchés urbains croissants, principalement le maraîchage, la banane, la pomme de terre, et les tubercules. Ces cultures sont devenues une source de revenus rendus indispensables par la marchandisation des moyens de production et des services agricoles (Sokoni, 2008) et également du fait de la faiblesse des alternatives non-agricoles dans les campagnes africaines.

Structuration d'un réseau marchand entre la campagne et la ville

Un ensemble de zones de production, de lieux et d'acteurs d'échanges se structure entre la campagne et la ville afin de fournir à cette dernière les productions alimentaires locales. Elles restent importantes dans les régimes alimentaires des urbains, les citadins demeurent attachés aux produits «du village ». Dans les montagnes d'Afrique de l'Est, cela se traduit par la densification du réseau des marchés qui ceinturent les massifs, la carte suivante indique les différentes périodes de création des places marchandes périodiques dans les montagnes Uporoto. 


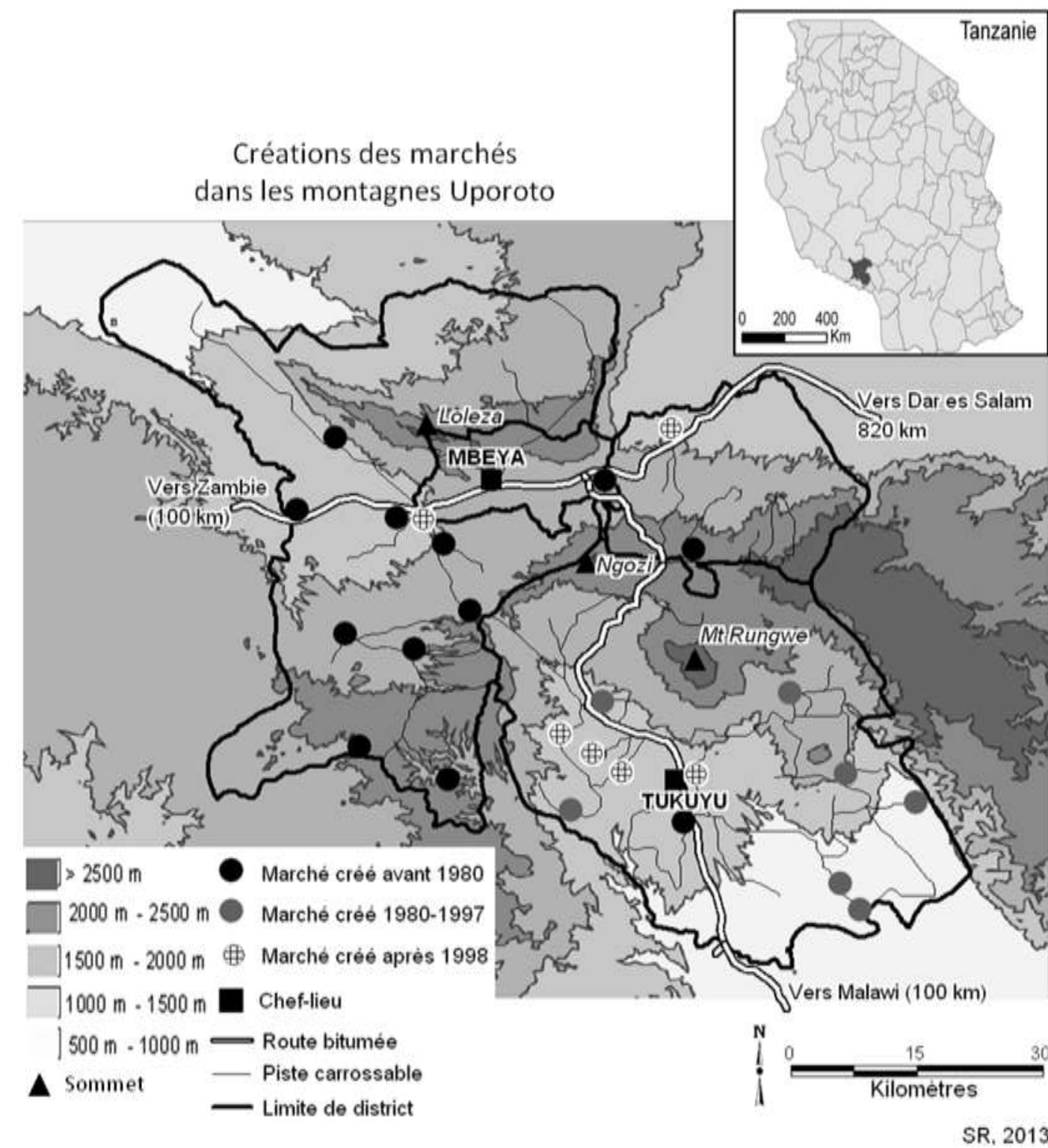

Figure 1 Création des marchés périodiques dans les montagnes Uporoto

Ces lieux de collecte et de redistribution ont une organisation hiérarchisée, ils font office d'interface entre le local, la métropole régionale et/ou les grandes villes nationales. Miroirs de leur arrière-pays, ils jouent un rôle structurant pour le monde rural en organisant les flux de produits bien qu'ils ne soient pas les lieux exclusifs des transactions.

Les intermédiaires privés ont investi ces filières à la fois dans le rural et dans l'urbain, ils assurent les liaisons entre les producteurs et les nombreux autres opérateurs dans le cadre d'un marché fragmenté. La dérégulation impose une situation dans laquelle l'accès aux intrants, au marché physique, et à l'information sur les prix, demeure problématique pour les paysans, par conséquent ils sont dans une position de faiblesse en matière de capacité de négociation.

Recompositions rurales liées à l'approvisionnement urbain

Ce modèle d'agriculture conduit à une spécialisation des terroirs et à leur mise en concurrence accrue à plusieurs échelles géographiques. Dans les montagnes Uporoto, entre 1800 et 2300 
mètres d'altitude, se constituent des espaces de production consacrés à la pomme de terre. Cette culture nécessite un capital important pour financer l'utilisation intensive d'engrais, de produits phytosanitaires et de main d'œuvre. Elle entretient une force de travail faiblement rémunérée à la tâche, des services à l'agriculture. La photographie suivante montre l'importance de la main d'œuvre mobilisée lors des récoltes de pommes de terre. Les nombreux ouvriers ont rempli les sacs qui seront chargés dans les camions à destination de Dar es Salam, la capitale économique du pays.

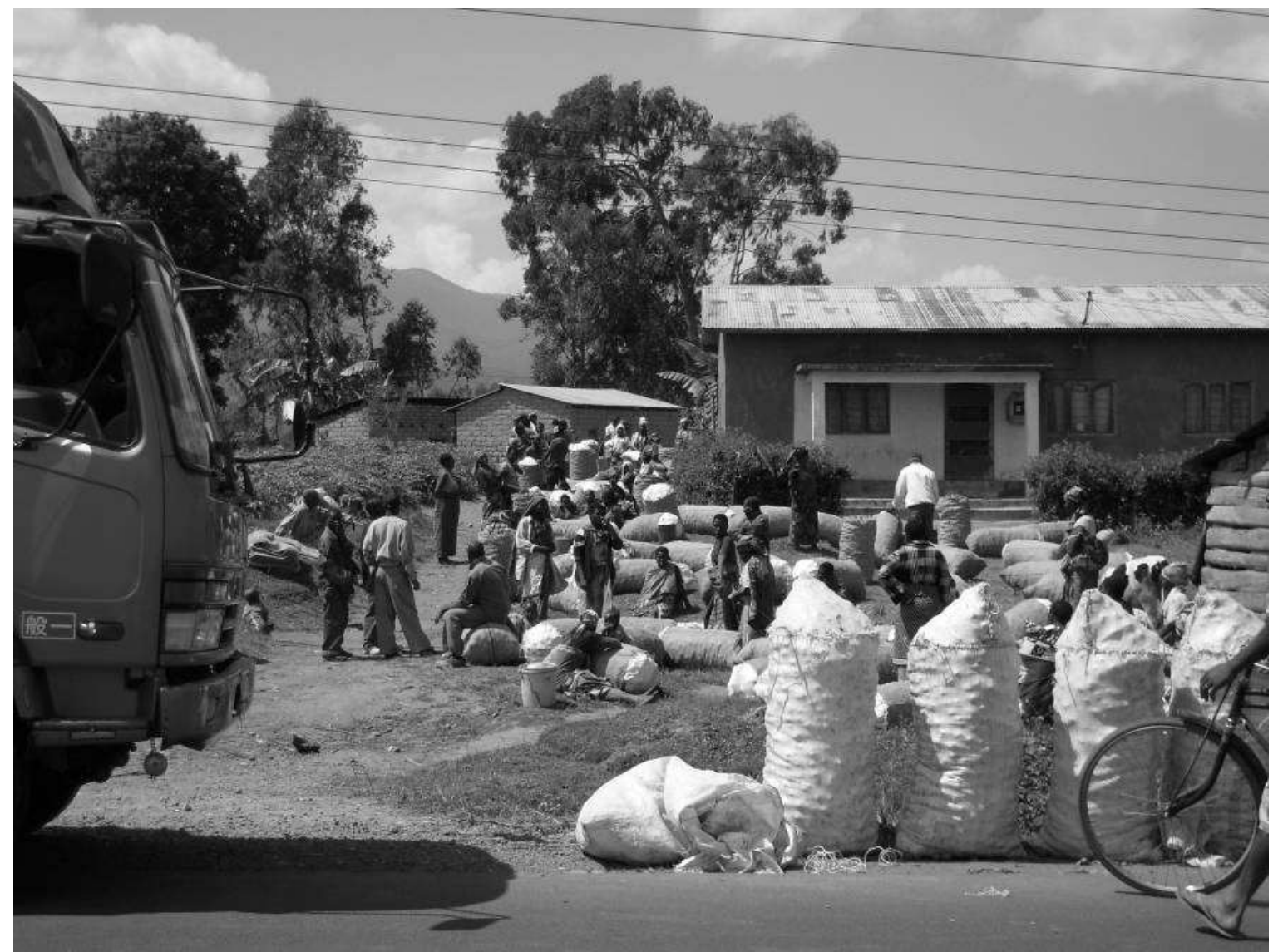

Figure 2 Main d'œuvre mobilisée pour la récolte et mise en sac de la pomme de terre, Uporoto Mountains (Racaud, 2011)

En outre, elle conduit à une compétition pour l'accès à la terre par des acteurs urbains qui cherchent à prendre à bail des parcelles le temps d'une ou de plusieurs récoltes. Le coût des intrants est régulièrement au dessus des moyens des paysans qui louent alors leurs parcelles qu'ils travaillent ensuite en tant qu'ouvrier, curieux paradoxe du propriétaire qui devient prolétaire sur sa terre. Le foncier devient un bien indifférencié de production et son exploitation intensive remet en cause sa fertilité qui était traditionnellement entretenue par des pratiques collectives.

L'approvisionnement des villes par l'agriculture de montagne conduit à une intégration de la paysannerie au marché. Ce modèle renforce les positions dominantes au sein des filières et il 
repose moins sur l'autonomie des paysans que sur leur domination ${ }^{4}$. De surcroît, l'essor de territoires marchands ne s'accompagne pas d'un véritable développement, au contraire, il favorise les contrastes territoriaux en fonction des différents avantages comparatifs (accessibilité, critères agro-écologiques, disponibilité des denrées, de la main d'œuvre) mobilisés pour le ravitaillement des marchés urbains. Pour autant, en l'absence d'alternative et en dépit des situations inéquitables, ce modèle apparaît la seule voie de développement, pour l'instant, fût-il inégal ou non durable.

Bibliographie :

Jean-Louis Chaleard, «Temps des villes, temps des vivres, l'essor du vivrier marchand en Côte d'Ivoire », Karthala, 1996.

Bernard Charlery de la Masseliere, «Le biais rural. Retour sur le développement », Revue Outre-Terre, 2005/2 nº11, pages 41 à 55, 2005.

Sylvain Racaud, Les montagnes Uporoto entre ville et campagne, géographie des flux et intégration territoriale en Tanzanie, Thèse de Géographie et Aménagement, Dynamiques Rurales, Université Toulouse 2-le Mirail, 2013.

Cosmas Sokoni, Commercialisation of smallholder production in Tanzania : implications for sustainable resources management, The Geographical Journal Volume $174 \mathrm{n}^{\circ} 2$, The Royal Geographical Society, 2008. URL : http://onlinelibrary.wiley.com/doi/10.1111/j.14754959.2008.00287.x/full

\footnotetext{
${ }^{4}$ Puisque les producteurs ont peu d'alternatives, peu de capacités de négociation et d'initiative, un accès au marché physique contraint par la faiblesse du capital et des infrastructures, et un accès à l'information dissymétrique révélateur de la position avantageuse des intermédiaires.
} 